\title{
Development and Evaluation of Antibody Proteomics Technology for Rapid and Comprehensive Identification of Potential Biomarkers and Therapeutic Targets
}

\author{
Kazuya Nagano \\ Laboratory of Toxicology and Safety Science, Graduate School of Pharmaceutical Sciences, \\ Osaka University; 1-6 Yamadaoka, Suita, Osaka 565-0871, Japan. \\ Received December 26, 2017
}

\begin{abstract}
Proteomics-based analyses are powerful means of identifying potentially useful proteins in the initial stage of drug development. Technological developments in the field of proteomics, and increases in the sensitivity of MS analyses, now facilitate identification and examination of increasingly small amounts of proteins that are differentially expressed in diseased versus normal tissues and can be candidate biomarkers or therapeutic targets. However, the current approach is for candidate proteins to be prioritized by research interest and then validated one by one; this is very inefficient. To address this issue, we have developed what we refer to as "antibody proteomics technology," which uses a phage antibody library and tissue microarray analysis to rapidly and comprehensively isolate monoclonal antibodies against candidate proteins for the identification of potential biomarkers and therapeutic targets. In our validation of this technology, we successfully identified oxysterol binding protein-like 5 and calumenin as potential biomarkers related to metastasis in lung cancer, annexin A4 as a potential biomarker related to cisplatin resistance in malignant mesothelioma, and Eph receptor $\mathrm{A} 10$ as a potential therapeutic target in breast cancer, including refractory breast cancer. These findings suggest that antibody proteomics technology has the potential to become a fundamental technology in drug discovery for the development of novel biomarkers and therapeutic targets.
\end{abstract}

Key words antibody proteomics technology; biomarker; therapeutic target; proteomics

\section{INTRODUCTION}

In the post-genome era, many of the proteins involved in pathological disorders and other biological processes are potential biomarkers or therapeutic targets. Therefore, many attempts are underway to develop a wide variety of moleculartargeted therapies and companion diagnostics for the treatment of intractable conditions such as cancers, infectious diseases, and autoimmune disorders. ${ }^{1,2)}$

Proteomics-based analyses are a powerful means of identifying potentially useful proteins in the initial stage of drug development, and this technology is now a major focus in drug discovery research., ${ }^{3,4)}$ Technological developments in the field of proteomics, and increases in the sensitivity of MS analyses, now mean that increasingly small amounts of proteins that are differentially expressed in diseased versus normal tissues can be identified and examined for their potential as candidate biomarkers or therapeutic targets. However, the current approach where candidate proteins are prioritized by research interest and are then validated one by one is very inefficient for identifying candidate proteins. Consequently, this approach has limited the number of proteins that have been identified and have progressed to development. Therefore, a means of comprehensively and analyzing the expression and function of many candidate proteins at once and to efficiently select potential proteins of interest is urgently needed. To address this issue, we have developed what we refer to as "antibody proteomics technology," which uses a phage antibody

This review of the author's work was written by the author upon receiving the 2017 Pharmaceutical Society of Japan Award for Young Scientists. library and tissue microarray analysis (TMA) to rapidly and comprehensively isolate monoclonal antibodies (mAbs) against candidate proteins for the identification of potential biomarkers and therapeutic targets. In this review, we describe the development and evaluation of this novel technology.

\section{ANTIBODY PROTEOMICS TECHNOLOGY: A HIGH-THROUGHPUT SYSTEM FOR VALIDATION OF MULTIPLE CANDIDATE PROTEINS}

Monoclonal antibodies and techniques such as enzymelinked immunosorbent assay, Western blotting, fluorescent imaging, and TMA are extremely useful for examining the function and distribution of proteins. ${ }^{5-7)}$ Usually, mAbs are generated by using hybridomas; however, this approach is laborious and time-consuming, and a large number of recombinant antigens is needed. Furthermore, protein production using this approach often requires gene engineering for heterologous expression, which takes time to optimize. Thus, it is impractical to produce mAbs against the many candidate proteins identified when using a proteomics approach to protein selection.

Previously, we constructed a phage antibody library comprising hundreds of millions of clones that allowed rapid isolation of mAbs against many target proteins in vitro within a span of two or three weeks. ${ }^{8)}$ Monoclonal antibodies can be isolated even from small amounts of antigen (only $0.5 \mathrm{ng}$ in the case of kinase insert domain receptor protein) by optimizing how they are selected (e.g., by using a nitrocellulose membrane panning method $)^{9}$ (Table 1). We therefore combined this phage antibody library with a proteomics approach and devel- 
Table 1. Comparison of Current Approaches for Preparing Monoclonal Antibodies

\begin{tabular}{|c|c|c|}
\hline Approach & Advantages & Disadvantages \\
\hline Hybridoma-based technology & $\begin{array}{l}\text { Easy to produce high-affinity mAbs via } \\
\text { immunization }\end{array}$ & $\begin{array}{l}\text { - Laborious and time-consuming } \\
\text { - Cumbersome when dealing with different types of antigen }\end{array}$ \\
\hline Naïve phage antibody library & $\begin{array}{l}\text { - Rapid and simple to construct and perform } \\
\text { - Easily deals with different types of antigen }\end{array}$ & - Difficult to isolate high-affinity mAbs \\
\hline $\begin{array}{l}\text { Naïve phage antibody library plus } \\
\text { nitrocellulose membrane panning }\end{array}$ & $\begin{array}{l}\text { - Rapid and simple to construct and perform } \\
\text { - Easily deals with different types of antigen } \\
\text { - Needs only small amounts of antigen }\end{array}$ & - Difficult to isolate high-affinity mAbs \\
\hline
\end{tabular}

oped what we refer to as "antibody proteomics technology," which allows us to simultaneously produce mAbs against many candidate proteins and to analyze the expression profiles and functions of these proteins in diseased versus normal tissues. ${ }^{9)}$ Thus, this technology accelerates the identification of proteins that are potentially useful as biomarkers or therapeutic targets. The antibody proteomics technology comprises four stages (Fig. 1): (1) a search for disease-related proteins by means of proteomics-based analysis using two-dimensional differential in-gel electrophoresis (2D-DIGE); (2) identification of the candidate proteins by means of MS analysis; (3) isolation of mAbs against the candidate proteins by using a phage antibody library; and (4) validation of the candidate proteins by using diseased and normal tissue samples by means of TMA. The advantages of this technology are that it allows high-throughput generation of mAbs against many candidate proteins by directly using small amounts of proteins extracted from the 2D-DIGE gel, thereby optimizing the mAb selection method, and also that it allows validation of candidate proteins by using tissue samples with various clinical characteristics such as different disease states, ages, or treatment histories. Therefore, by analyzing the correlation between expression profile and clinical information, each candidate protein can be comprehensively validated.

\section{DISCOVERY OF NOVEL BIOMARKERS AND DRUG TARGETS THROUGH VALIDATION OF THE ANTIBODY PROTEOMICS TECHNOLOGY}

To validate the usefulness of the antibody proteomics technology, we used the technology to identify several novel biomarkers and drug targets in three different disease states. Here, we introduce two potential biomarkers related to metastasis in lung cancer, ${ }^{10,11)}$ one potential biomarker related to cisplatin resistance in malignant mesothelioma, ${ }^{12}$ and one potential therapeutic target in breast cancer, including refractory breast cancer ${ }^{13-15)}$ that were discovered by using the antibody proteomics technology.

3.1. Search for a Biomarker Related to Metastasis in Lung Cancer Lung cancer is the leading cause of cancer-related death worldwide; it accounts for $17.6 \%$ of all deaths from cancer and it has a five-year survival rate of only $8.9-15 \%{ }^{16)}$ Metastasis is a poor prognosis factor in lung cancer, and is the main cause of treatment failure and death. ${ }^{17)}$ Therefore, preventing metastasis is an important step to decrease lung cancer-related mortality. Several mechanistic studies have revealed that various proteases ${ }^{18)}$ and chaperones, ${ }^{19)}$ as well as the epithelial-mesenchymal transition ${ }^{20)}$ and lipid metabolism, ${ }^{21)}$ may be involved in metastasis. However, these results are not conclusive, and no promising candidate proteins have yet been identified for the accurate diagnosis, prediction, and

\section{Biography}

Kazuya Nagano was born in Hokkaido in 1980. He graduated from the School of Pharmaceutical Sciences, University of Shizuoka, in 2005, and completed a master's degree from the Graduate School of Pharmaceutical Sciences, Osaka University in 2007. In 2007, he began his research career as a research fellow with a Research Fellowship for Young Scientists from the Japan Society for the Promotion of Science (DC1<doctoral course 1>). Nagano was then appointed as a research fellow with the Laboratory of Pharmaceutical Proteomics Project, National Institute of Biomedical Innovation (under Project Leader Yasuo Tsutsumi), from 2009 to 2010, and subsequently with the Laboratory of Biopharmaceutical Research, National Institute of Biomedical Innovation (under Project Leader Shin-ichi Tsunoda) from 2010 to 2015. He was also appointed as a visiting researcher at the Graduate School of Pharmaceutical Sciences, Osaka University, from 2013 to 2015, and received his

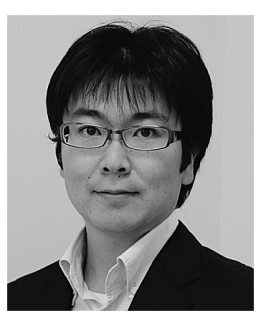

Kazuya Nagano Ph.D. degree from Osaka University in 2014. Currently, Dr. Nagano is an associate professor in the Laboratory of Toxicology and Safety Science at the Graduate School of Pharmaceutical Sciences, Osaka University (under Professor Yasuo Tsutsumi). Throughout his career, Dr. Nagano has searched for drug targets or drug seeds in an attempt to develop novel antibody drugs. His work has focused primarily on the development of an antibody proteomics system. As a result of his research, Dr. Nagano has received various awards: from the University of Shizuoka (2005), the Kinki Branch of the Pharmaceutical Society of Japan (2009), the Japanese Association for Metastasis Research (2010), the Japan Society of Drug Delivery Systems (2012), the Clinical Pharmaceutical Sciences Division of the Pharmaceutical Society of Japan (2014), and the Pharmaceutical Society of Japan (2017). 


\section{Usual proteomics approach}

\section{1) 2D-DIGE analysis and spot picking}

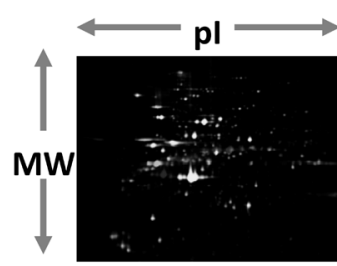

2) Identification of proteins by MS analysis

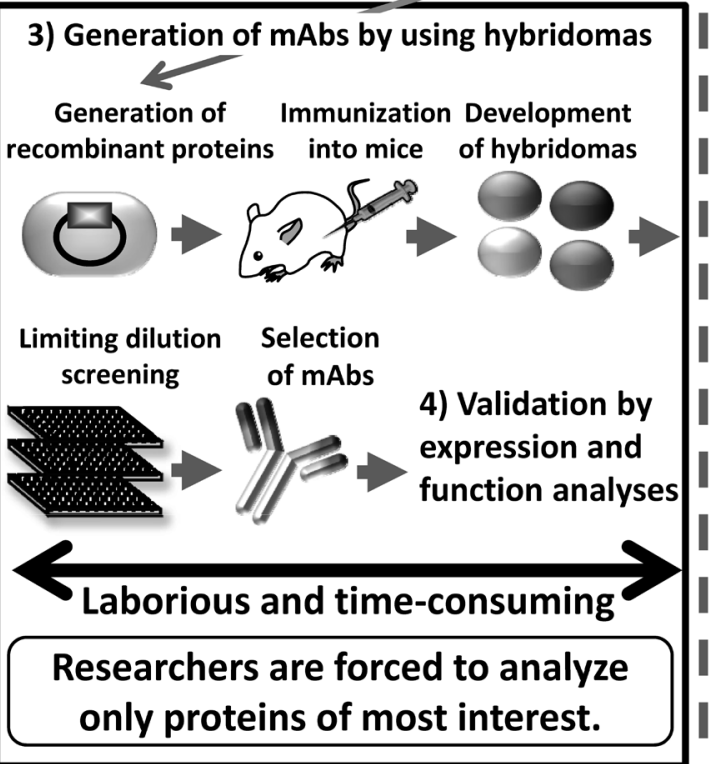

\section{Antibody proteomics technology}

\section{1) 2D-DIGE analysis and spot picking}

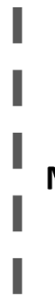

\section{2) Identification of proteins by MS analysis}

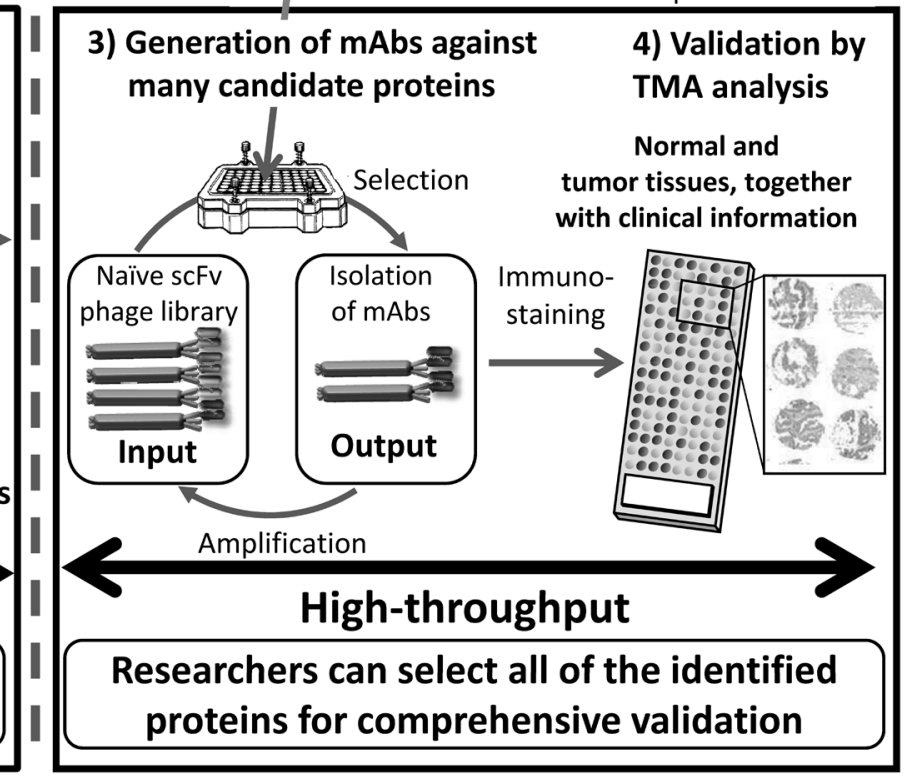

Fig. 1. Comparison of Protein Identification and Validation by Means of the Usual Proteomics Approach and the Antibody Proteomics Technology Approach

The usual proteomics approach (left panel) in which candidate proteins are prioritized by research interest and are then validated one by one is very inefficient for identifying potential biomarkers and therapeutic targets. To address this issue, we developed a high-throughput system for validation of disease-related proteins, which we call "antibody proteomics technology" (right panel): 1) Candidate proteins are detected by using two-dimensional differential in-gel electrophoresis (2D-DIGE) and 2) identified by means of MS analysis. At the same time, 3) monoclonal antibodies (mAbs) against all of the proteins identified by 2D-DIGE are produced by using a phage antibody library. Finally, 4) the proteins are validated as biomarkers and drug targets by means of tissue microarray analysis (TMA). Thus, by using this technology, all candidate proteins can be comprehensively validated and the most useful proteins selected. (Modified and recreated from Ref. 9.)

regulation of lung cancer metastasis in the clinical setting. Therefore, we used our antibody proteomics technology to identify novel biomarkers related to metastasis in lung cancer.

To identify metastasis-related proteins in lung cancer, we performed a 2D-DIGE analysis of two human lung adenocarcinoma cell lines, one with high lymph node metastatic potential (RERF-LC-KJ) ${ }^{22)}$ and one with no metastatic potential (RERF-LC-MS) ${ }^{23)}$ As a result of the 2D-DIGE analysis, 15 proteins with more than a two-fold difference in intensity between the RERF-LC-KJ and RERF-LC-MS cells were found and then identified by means of MS analysis. We then isolated mAbs against each identified protein by using our phage antibody library. Nitrocellulose membrane panning showed that several mAbs bound to each of the 15 identified proteins. From these mAbs, we selected those with the highest affinities and specificities for their respective target proteins.

To select the proteins specifically related to lung cancer metastasis from the 15 candidate proteins, the expression profiles of the candidate proteins were determined by means of TMA with the isolated mAbs. The TMA analysis revealed that among the candidate proteins, the expression ratios of oxys- terol binding protein-like 5 (OSBPL5) and calumenin (CALU) were significantly higher in lymph node metastasis-positive tissue samples than in metastasis-negative tissues samples ( $p=0.0156, p=0.0055$, respectively) (Fig. 2). Moreover, 15 tissue samples among a total of 46 lung tumor samples were OSBPL5 and CALU double-positive, and 12 of these $15(80 \%)$ were lymph node metastasis-positive. Therefore, the correlation analysis between protein expression and clinicopathological characteristics revealed a significant association between OSBPL5 and CALU expression and lymph node metastasis in lung adenocarcinoma.

Finally, we examined the effects of gene over-expression and gene knockdown on the invasiveness, an important trait for metastasis, of the two lung cancer cell lines to delineate the functions of OSBPL5 and CALU in metastatic lung cancer. An in vitro invasion assay showed that RERF-LC-MS cells overexpressing OSBPL5 or CALU were significantly more invasive than cells transfected with a control plasmid, and that the invasiveness of RERF-LC-KJ cells transfected with OSBPL5 or CALU small interfering RNA (siRNA) was significantly lower compared than that of cells transfected 


\section{Lymph node metastasis-negative tissue samples}
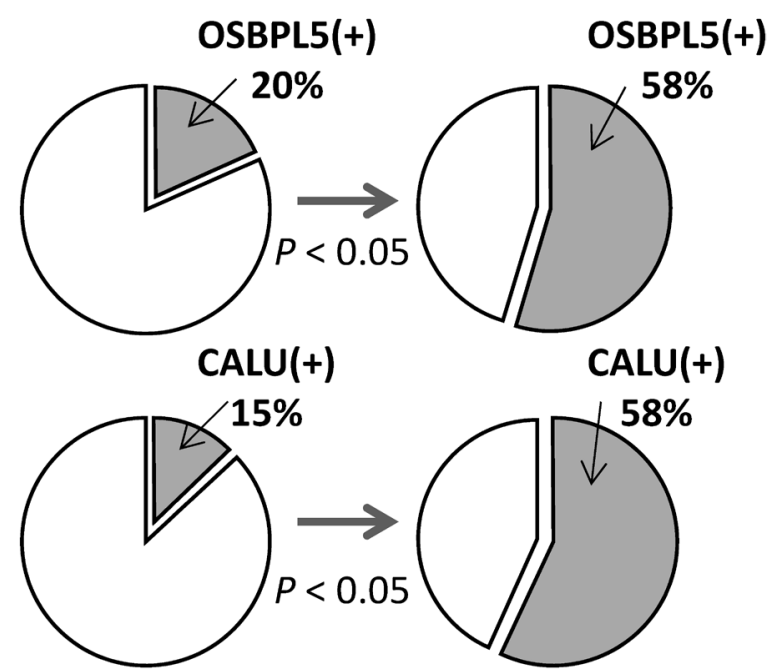

Fig. 2. Expression of Oxysterol Binding Protein-Like 5 (OSBPL5) or Calumenin (CALU) Is Associated with Lymph Node Metastasis in Lung Cancer

A tissue microarray analysis slide containing 46 samples of lung cancer tissue (20 samples of lymph node metastasis-positive tissue; 26 samples of lymph node metastasis-negative tissue) was immunostained with an anti-OSBPL5 monoclonal antibody and an anti-CALU monoclonal antibody isolated from a phage antibody library. The proportion of tissues that were OSBPL5- or CALU-positive was significantly larger in lymph node metastasis-positive tissues compared with in lymph node metastasis-negative tissues $(p<0.05$; differences compared with the MannWhitney $U$ test). (Modified and recreated from Ref. 10.)

with a control plasmid.

These results suggest that OSBPL5 and CALU may play a critical role in determining the invasiveness of lung adenocarcinoma cells. ${ }^{10)}$ We hope that the data obtained from this study will contribute to elucidation of the molecular mechanisms underlying metastasis and help in the development of diagnostic markers and drugs against lung cancer metastasis.

3.2. Search for a Biomarker Related to Cisplatin-Resistance in Malignant Mesothelioma Malignant mesothelioma is an aggressive cancer of serous membranes such as the pleura. This type of cancer has a poor prognosis, and the fiveyear survival rate is only $5 \% .{ }^{24,25)}$ An increase in mesothelioma patients is expected in the near future because malignant mesothelioma takes $40-50$ years to develop after exposure to asbestos, which was a commonly used building material in the 1960-70s. Cisplatin is a first-line anticancer drug for the treatment of malignant mesothelioma; however, only about $20 \%$ of patients respond. ${ }^{26,27)}$ In addition, a major problem with cisplatin treatment of malignant mesothelioma is the development of cisplatin resistance. Therefore, it is important not only to develop novel means of distinguishing responders from nonresponders but also to elucidate the molecular mechanisms underlying the development of cisplatin resistance. Therefore, we conducted an antibody proteomics analysis by using highly or slightly cisplatin-susceptible malignant mesothelioma cells to identify proteins associated with cisplatin susceptibility.

We first obtained highly and slightly cisplatin-susceptible malignant mesothelioma cells. Of five mesothelioma cell lines tested (H28, H2052, H2452, H226, and MSTO-221H), cisplat-

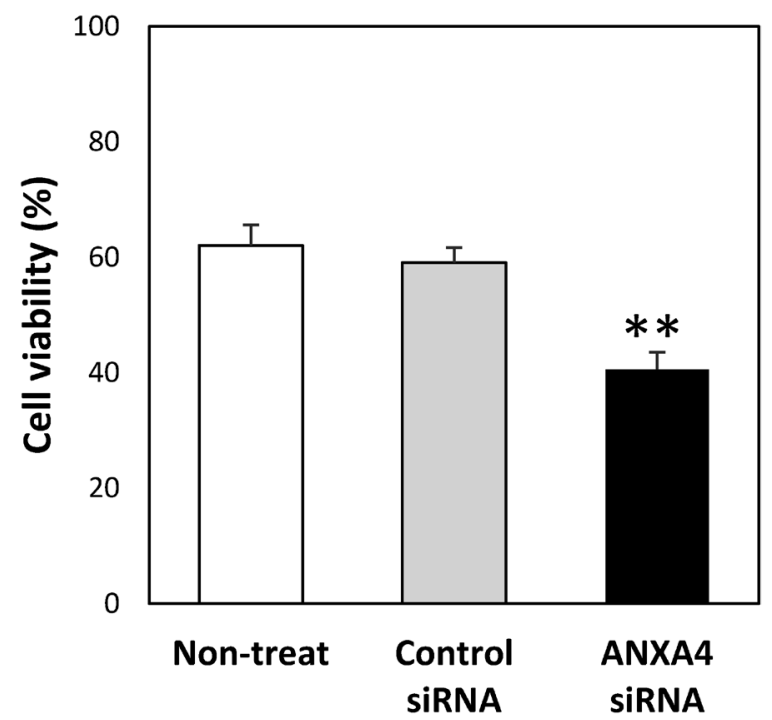

Fig. 3. Expression of Annexin A4 Regulates Cisplatin Susceptibility in Malignant Mesothelioma

To evaluate the function of annexin A4 (ANXA4), a highly expressed protein in H28 malignant mesothelioma cells with low cisplatin susceptibility, cisplatin susceptibility was assayed by using a genetic engineering approach. A WST-8 assay showed that cisplatin susceptibility was significantly increased in malignant mesothelioma cells transfected with small interfering RNA (siRNA) silencing the expression of genes encoding ANXA4. Results are expressed as mean \pm standard deviation (S.D.) (cisplatin conc., $40 \mu \mathrm{M} ; n=3 ; * *, p<0.01$ compared with the value for the control siRNA-treated group; differences compared by using one-way ANOVA with Bonferroni's test). (Modified and recreated from Ref. 12.)

in susceptibility was highest in $\mathrm{H} 2052$ cells and lowest in $\mathrm{H} 28$ cells (data not shown). A 2D-DIGE analysis afforded eight proteins with more than a two-fold difference in expression between $\mathrm{H} 28$ and $\mathrm{H} 2052$ cells, and these proteins were then identified by means of MS analysis. Next, we isolated mAbs against each candidate protein by using our phage antibody library and then conducted a TMA using malignant mesothelioma tissues, benign mesothelioma tissues, and normal mesothelial tissues; we found that annexin A4 (ANXA4) expression was higher in malignant mesothelioma tissues than in benign mesothelioma tissues and normal mesothelial tissues.

We next evaluated the involvement of ANXA4 in cisplatin susceptibility by using a genetic engineering approach. To evaluate the correlation between ANXA4 expression and cisplatin susceptibility, ANXA4-siRNA and ANXA4-pcDNA3.1, a mammalian expression vector, were transfected to $\mathrm{H} 28$ and $\mathrm{H} 2052$ cells, respectively, before cisplatin treatment. The $\mathrm{IC}_{50}$ values of non-transfected $\mathrm{H} 28$ cells, or of $\mathrm{H} 28$ cells transfected with either control-siRNA or ANXA4-siRNA were $80.0,71.8$, and $15.5 \mu \mathrm{M}$, respectively, and those of $\mathrm{H} 2052$ cells transfected with either control-pcDNA3.1 or ANXA4pcDNA3.1 were 55.2 and $89.7 \mu \mathrm{M}$, respectively (Fig. 3). These data indicated that the cisplatin susceptibility of $\mathrm{H} 28$ cells was increased by ANXA4-siRNA transfection and that of H2052 cells was decreased by ANXA4-pcDNA 3.1 transfection, suggesting that ANXA4 was associated with cisplatin resistance in mesothelioma cells and is therefore a potential biomarker for distinguishing cisplatin responders from non-responders. ${ }^{12)}$

We hope that these findings will contribute to the elucidation of the molecular mechanisms underlying cisplatin resistance in the treatment of malignant mesothelioma and help in developing companion diagnostics for the use of cisplatin. 
Table 2. Expression of Eph Receptor A10 (EphA10) in Four Subtypes of Breast Cancer

\begin{tabular}{|c|c|c|c|c|c|}
\hline \multirow{2}{*}{ Subtype name } & \multicolumn{2}{|c|}{ Expression } & \multirow{2}{*}{$\begin{array}{l}\text { Numbers of EphA10-positive } \\
\text { tissue samples (Ratio) }\end{array}$} & \multirow{2}{*}{$\begin{array}{l}\text { Numbers of EphA10-negative } \\
\text { tissue samples (Ratio) }\end{array}$} & \multirow{2}{*}{ Total } \\
\hline & Her2 & ER PR & & & \\
\hline Luminal A & - & + & $22(54 \%)$ & $19(46 \%)$ & 41 \\
\hline Luminal B & + & + & $25(68 \%)$ & $12(32 \%)$ & 37 \\
\hline Her2-enriched & + & - & $16(64 \%)$ & $11(36 \%)$ & 27 \\
\hline TNBC & - & - & $10(67 \%)$ & $5(33 \%)$ & 15 \\
\hline Total & & & $73(61 \%)$ & $47(39 \%)$ & 120 \\
\hline
\end{tabular}

(Modified and recreated from Ref. 14.)

3.3. Search for a Drug Target in Breast Cancer, Including Refractory Breast Cancer Her2-targeting therapies such as trastuzumab, and hormone therapies such as tamoxifen, are effective for the treatment of breast cancer, and the use of these drugs has improved the prognosis of patients with breast cancer. ${ }^{28)}$ However, Her2 is only expressed in $20-30 \%$ of breast cancers, which limits the therapeutic utility of antiHer2 antibodies. ${ }^{28)}$ In addition, many patients given long-term Her2-targeting therapy and hormone treatment eventually develop resistance, even if the cancers being treated are Her2and/or hormone receptor-positive. ${ }^{28)}$ Also, it is difficult to use hormone therapies simultaneously with Her2-targeting therapy in triple-negative breast cancer, which does not express Her2, estrogen receptor (ER), or progesterone receptor (PR). ${ }^{29)}$ Thus, novel therapeutic targets for the treatment of breast cancer are urgently needed. We therefore used our antibody proteomics technology to search for potential therapeutic targets by comparing the protein expression profiles of SKBR3 breast cancer cells and 184A1 normal cells.

By using 2D-DIGE and MS analyses, we identified 21 proteins differentially expressed in the breast cancer cells. Monoclonal antibodies against the 21 candidate proteins were then successfully isolated by using our phage antibody library. TMA analysis showed that cytokeratin 8 , tumor necrosis factor-related apoptosis-inducing ligand receptor 2 (TRAIL-R2), and Eph receptor A10 (EphA10) were specifically expressed in clinical samples of breast tumor tissues. ${ }^{9)}$ Cytokeratin 8 and TRAIL-R2 are well-known biomarkers and therapeutic targets. However, EphA10 is a relatively uncharacterized protein, although it is reported to be expressed in the testes. Therefore, we evaluated EphA10 as a potential drug target by determining its protein expression profile and by administering an antiEphA10 antibody to mice bearing EphA10-expressing tumor.

Breast cancers can be divided into four therapeutically relevant subtypes on the basis of tumor cell Her2, ER, and PR expression: luminal A (Her2 ${ }^{-}, \mathrm{ER}^{+}$, and/or $\mathrm{PR}^{+}$), luminal $\mathrm{B}$ $\left(\mathrm{Her}^{+}, \mathrm{ER}^{+}\right.$, and/or $\left.\mathrm{PR}^{+}\right)$, Her2-enriched $\left(\mathrm{Her} 2^{+}, \mathrm{ER}^{-}, \mathrm{PR}^{-}\right.$), and triple-negative $\left(\mathrm{Her} 2^{-}, \mathrm{ER}^{-}, \mathrm{PR}^{-}\right){ }^{30)}$ To comprehensively evaluate the utility of EphA10 as a therapeutic target for the treatment of breast cancer, we first analyzed its expression in the above-mentioned subtypes by means of TMA using breast cancer tissues with Her2, ER and PR expression profile. EphA10 was found to be expressed by all four subtypes of cancer cells. Further immunohistochemical analyses indicated that the proportion of EphA10-positive cases per subtype was as follows: luminal A (54\%), luminal B (68\%), Her2-enriched (64\%), and triple-negative (67\%) (Table 2). These data suggest that EphA10 may be an attractive therapeutic target for the treatment of breast cancer, particularly in patients with triple-negative breast cancer, who are under-served by current molecular-targeted drugs.

To evaluate the specificity of EphA10 expression, we next conducted a TMA of EphA10 levels in 36 different types of normal (non-cancerous) tissues, which confirmed that EphA10 was expressed in the testes, but not in any of the other 35 kinds of tissues tested. We also confirmed the testis-specific expression profile of EphA10 at the mRNA level via realtime quantitative PCR. These data suggested that EphA10 is a highly specific tumor antigen and is therefore a potentially useful therapeutic target for the treatment of breast cancer, including triple-negative type.

To evaluate the utility of EphA10 as a therapeutic target in more detail, we developed an anti-EphA10 mAb and determined in vivo distribution and anti-tumor effects in a xenograft mouse model.

First, we assessed the distribution of the anti-EphA10 mAb in xenograft mice bearing EphA10-expressing tumors. Alexa Fluor 647-labeled anti-EphA10 mAb or control mAb were intravenously injected into the mice. At four days after administration, in vivo imaging revealed that fluorescence was concentrated in the tumor tissues of anti-EphA10 mAb-treated mice but not in those of the control mAb-treated mice. Furthermore, the mean fluorescent intensity of tumor tissues isolated from the mice at day 4 was significantly stronger in the tissues from the anti-EphA10 mAb-treated mice than in those from the control mAb-treated mice $(p<0.05)$. These results indicate that the anti-EphA10 mAb specifically accumulated in EphA10-expressing tumor tissues, indicating its potential as a tool for targeting breast cancer tumor tissue.

To evaluate the biological effects of accumulation of antiEphA10 mAb in tumor tissue, we next intraperitoneally administered anti-EphA10 mAb (1 or $0.5 \mathrm{mg} / \mathrm{mouse}$ ) or control $\mathrm{mAb}(1 \mathrm{mg} / \mathrm{mouse})$ and examined tumor growth over time. On the basis of tumor tissue volume measurements, tumor growth was significantly suppressed in anti-EphA10 mAb-treated mice compared with in control mice, with this effect occurring in a dose-dependent manner. At day 47 after administration of the anti-EphA10 mAb, we isolated tumor masses from the mice and measured tumor weight. In agreement with the volumetric analysis, the tumor tissue weights were less for the anti-EphA10 mAb-treated mice, with this effect again occurring in a dose-dependent manner. Since the bodyweight of the mice in the different groups was comparable at the time of administration of the antibody, indirect effects of $\mathrm{mAb}$ administration on bodyweight loss were ruled out. Thus, the $\mathrm{mAb}$ accumulated in EphA10-expressing tumor tissue and significantly suppressed growth without obvious adverse effects. These findings suggest that EphA10 is a potentially promising 
therapeutic target for the treatment of breast cancers, including triple-negative type. ${ }^{14)}$

\section{CONCLUSION}

Here, we reviewed our progress in addressing the bottleneck in the proteomics approach to identifying proteins potentially useful as biomarkers or therapeutic targets, which is the inefficacy of current methods for producing and then validating $\mathrm{mAbs}$ against multiple candidate proteins. To address this bottleneck, we developed an approach that we refer to as "antibody proteomics technology," which uses a phage antibody library and TMA to simultaneously screen multiple proteins by isolating $\mathrm{mAbs}$ against each candidate in a rapid, comprehensive manner. Through our validation of the antibody proteomics technology, we successfully identified two potential biomarkers related to metastasis in lung cancer, a potential biomarker related to cisplatin-resistance in malignant mesothelioma, and a potential therapeutic target in breast cancer, including in refractory breast cancer.

Recent developments in the field of molecular-targeted drugs have improved the prognosis of patients with intractable diseases, such as cancers and autoimmune diseases, and many molecular-targeted drug candidates are undergoing evaluation in clinical trials. However, the overall number of therapeutic targets is limited, so the development of best-in-class drugs is currently the major development strategy adopted by pharmaceutical companies. We hope that adoption of our antibody proteomics technology will provide many more novel therapeutic targets and promote the development of more first-inclass drugs.

Acknowledgments This study was supported, in part, by Grants-in-Aid for Scientific Research from the Ministry of Education, Culture, Sports, Science and Technology of Japan, the Japan Science and Technology Agency (JST), and the Japan Society for the Promotion of Science (JSPS); by a Grant-in-Aid for JSPS Fellows; and by Health Labour Sciences Research Grants from the Ministry of Health, Labour and Welfare of Japan.

Conflict of Interest The author declares no conflict of interests.

\section{REFERENCES}

1) Evans WE, Relling MV. Moving towards individualized medicine with pharmacogenomics. Nature, 429, 464-468 (2004).

2) Plenge RM, Scolnick EM, Altshuler D. Validating therapeutic targets through human genetics. Nat. Rev. Drug Discov., 12, 581-594 (2013).

3) Hanash S. Disease proteomics. Nature, 422, 226-232 (2003).

4) Kavallaris M, Marshall GM. Proteomics and disease: opportunities and challenges. Med. J. Aust., 182, 575-579 (2005).

5) Kaufmann H, Bailey JE, Fussenegger M. Use of antibodies for detection of phosphorylated proteins separated by two-dimensional gel electrophoresis. Proteomics, 1, 194-199 (2001).

6) $\mathrm{Au} \mathrm{NH}$, Gown AM, Cheang M, Huntsman D, Yorida E, Elliott WM, Flint J, English J, Gilks CB, Grimes HL. P63 expression in lung carcinoma: a tissue microarray study of 408 cases. Appl. Immunohistochem. Mol. Morphol., 12, 240-247 (2004).

7) Rimm DL, Camp RL, Charette LA, Costa J, Olsen DA, Reiss M.
Tissue microarray: a new technology for amplification of tissue resources. Cancer J., 7, 24-31 (2001).

8) Imai S, Mukai Y, Nagano K, Shibata H, Sugita T, Abe Y, Nomura T, Tsutsumi Y, Kamada H, Nakagawa S, Tsunoda S. Quality enhancement of the non-immune phage $\mathrm{scFv}$ library to isolate effective antibodies. Biol. Pharm. Bull., 29, 1325-1330 (2006).

9) Imai S, Nagano K, Yoshida Y, Okamura T, Yamashita T, Abe Y, Yoshikawa T, Yoshioka Y, Kamada H, Mukai Y, Nakagawa S, Tsutsumi Y, Tsunoda S. Development of an antibody proteomics system using a phage antibody library for efficient screening of biomarker proteins. Biomaterials, 32, 162-169 (2011)

10) Nagano $K$, Imai $S$, Zhao $X$, Yamashita $T$, Yoshioka $Y$, Abe $Y$, Mukai Y, Kamada H, Nakagawa S, Tsutsumi Y, Tsunoda S. Identification and evaluation of metastasis-related proteins, oxysterol binding protein-like 5 and calumenin, in lung tumors. Int. J. Oncol., 47, 195-203 (2015)

11) Yamashita $T$, Okamura $T$, Nagano $K$, Imai $S$, Abe $Y$, Nabeshi $H$, Yoshikawa T, Yoshioka Y, Kamada H, Tsutsumi Y, Tsunoda S. Rho GDP-dissociation inhibitor alpha is associated with cancer metastasis in colon and prostate cancer. Pharmazie, 67, 253-255 (2012).

12) Yamashita $T$, Nagano $K$, Kanasaki S, Maeda $Y$, Furuya $T$, Inoue M, Nabeshi H, Yoshikawa T, Yoshioka Y, Itoh N, Abe Y, Kamada H, Tsutsumi Y, Tsunoda S. Annexin A4 is a possible biomarker for cisplatin susceptibility of malignant mesothelioma cells. Biochem. Biophys. Res. Commun., 421, 140-144 (2012).

13) Nagano K, Kanasaki S, Yamashita T, Maeda Y, Inoue M, Higashisaka K, Yoshioka Y, Abe Y, Mukai Y, Kamada H, Tsutsumi Y, Tsunoda S. Expression of Eph receptor A10 is correlated with lymph node metastasis and stage progression in breast cancer patients. Cancer Med., 2, 972-977 (2013).

14) Nagano $K$, Maeda $Y$, Kanasaki $S$, Watanabe $T$, Yamashita $T$, Inoue M, Higashisaka K, Yoshioka Y, Abe Y, Mukai Y, Kamada H, Tsutsumi Y, Tsunoda S. Ephrin receptor A10 is a promising drug target potentially useful for breast cancers including triple negative breast cancers. J. Control. Release, 189, 72-79 (2014).

15) Nagano K, Yamashita $T$, Inoue M, Higashisaka K, Yoshioka $Y$, Abe Y, Mukai Y, Kamada H, Tsutsumi Y, Tsunoda S. Eph receptor A10 has a potential as a target for a prostate cancer therapy. Biochem. Biophys. Res. Commun., 450, 545-549 (2014).

16) Parkin DM, Bray F, Ferlay J, Pisani P. Global cancer statistics, 2002. CA Cancer J. Clin., 55, 74-108 (2005).

17) Steeg PS. Metastasis suppressors alter the signal transduction of cancer cells. Nat. Rev. Cancer, 3, 55-63 (2003).

18) Rochefort H, Capony F, Garcia M. Cathepsin D: a protease involved in breast cancer metastasis. Cancer Metastasis Rev., 9, 321-331 (1990).

19) Koga F, Kihara K, Neckers L. Inhibition of cancer invasion and metastasis by targeting the molecular chaperone heat-shock protein 90. Anticancer Res., 29, 797-807 (2009).

20) Kang Y, Massague J. Epithelial-mesenchymal transitions: twist in development and metastasis. Cell, 118, 277-279 (2004).

21) Torosian M, Charland S, Lappin J. Biochemical modulation of tumor-growth, metastasis and host metabolism. Oncol. Rep., 2, 1141-1145 (1995).

22) Teraoka S, Kyoizumi S, Seyama T, Yamakido M, Akiyama M. Scid mice model for the in-vivo study of human oncotherapy-studies on the growth and metastasis of human lung-cancer. Int. J. Oncol., 5, 501-508 (1994)

23) Hirai K, Shimada H, Ogawa T, Taji S. The spread of human lung cancer cells on collagens and its inhibition by type III collagen. Clin. Exp. Metastasis, 9, 517-527 (1991).

24) Heintz NH, Janssen-Heininger YM, Mossman BT. Asbestos, lung cancers, and mesotheliomas: from molecular approaches to targeting tumor survival pathways. Am. J. Respir. Cell Mol. Biol., 42, 133-139 (2010).

25) Rom WN, Travis WD, Brody AR. Cellular and molecular basis of 
the asbestos-related diseases. Am. Rev. Respir. Dis., 143, 408-422 (1991).

26) Berghmans T, Paesmans M, Lalami Y, Louviaux I, Luce S, Mascaux C, Meert AP, Sculier JP. Activity of chemotherapy and immunotherapy on malignant mesothelioma: a systematic review of the literature with meta-analysis. Lung Cancer, 38, 111-121 (2002).

27) Mintzer DM, Kelsen D, Frimmer D, Heelan R, Gralla R. Phase II trial of high-dose cisplatin in patients with malignant mesothelioma. Cancer Treat. Rep., 69, 711-712 (1985).

28) Lin SX, Chen J, Mazumdar M, Poirier D, Wang C, Azzi A, Zhou
M. Molecular therapy of breast cancer: progress and future directions. Nat. Rev. Endocrinol., 6, 485-493 (2010).

29) Carey L, Winer E, Viale G, Cameron D, Gianni L. Triple-negative breast cancer: disease entity or title of convenience? Nat. Rev. Clin. Oncol., 7, 683-692 (2010).

30) Huber KE, Carey LA, Wazer DE. Breast cancer molecular subtypes in patients with locally advanced disease: impact on prognosis, patterns of recurrence, and response to therapy. Semin. Radiat. Oncol., 19, 204-210 (2009) 